\title{
Cortical dopaminergic innervation among humans, chimpanzees, and macaque monkeys: A comparative study
}

\author{
Mary Ann Raghanti ${ }^{1,{ }^{*}}$, Cheryl D. Stimpson ${ }^{2}$, Jennifer L. Marcinkiewicz ${ }^{3}$, Joseph M. Erwin ${ }^{4}$, \\ Patrick R. Hof ${ }^{5,6}$, and Chet C. Sherwood ${ }^{2}$ \\ ${ }^{1}$ Department of Anthropology and School of Biomedical Sciences, Kent State University, Kent, \\ $\mathrm{OH}$ \\ ${ }^{2}$ Department of Anthropology, The George Washington University, Washington, D.C. \\ ${ }^{3}$ Biological Sciences, Kent State University, Kent, $\mathrm{OH}$ \\ ${ }^{4}$ Department of Biomedical Sciences and Pathobiology, Virginia-Maryland Regional College of \\ Veterinary Medicine, Virginia Tech, Blacksburg, VA \\ ${ }^{5}$ Department of Neuroscience, Mount Sinai School of Medicine, New York, NY \\ ${ }^{6}$ New York Consortium in Evolutionary Primatology, New York, NY
}

\begin{abstract}
In this study, we assessed the possibility that humans differ from other primate species in the supply of dopamine to the frontal cortex. To this end, quantitative comparative analyses were performed among humans, chimpanzees, and macaques using immunohistochemical methods to visualize tyrosine hydroxylase-immunoreactive axons within the cerebral cortex. Axon densities and neuron densities were quantified using computer-assisted stereology. Areas 9 and 32 were chosen for evaluation due to their roles in higher-order executive functions and theory of mind, respectively. Primary motor cortex (area 4) was also evaluated because it is not directly associated with cognition. We did not find an overt quantitative increase in cortical dopaminergic innervation in humans relative to the other primates examined. However, several differences in cortical dopaminergic innervation were observed among species which may have functional implications. Specifically, humans exhibited a sublaminar pattern of innervation in layer I of areas 9 and 32 that differed from that of macaques and chimpanzees. Analysis of axon length density to neuron density among species revealed that humans and chimpanzees together deviated from macaques in having increased dopaminergic afferents in layers III and V/VI of areas 9 and 32, but there were no phylogenetic differences in area 4. Finally, morphological specializations of axon coils that may be indicative of cortical plasticity events were observed in humans and chimpanzees, but not macaques. Our findings suggest significant modifications of dopamine's role in cortical organization occurred in the evolution of the apes, with further changes in the descent of humans.
\end{abstract}

\section{Keywords}

tyrosine hydroxylase; prefrontal cortex; area 9; area 32; area 4; human evolution

(C) 2008 IBRO. Published by Elsevier Ltd. All rights reserved.

*Corresponding author: Department of Anthropology Lowry Hall Kent State University Kent, Ohio 44242 Phone: 330-672-9354 Fax: 330-672-2999 mraghant@kent.edu .

Publisher's Disclaimer: This is a PDF file of an unedited manuscript that has been accepted for publication. As a service to our customers we are providing this early version of the manuscript. The manuscript will undergo copyediting, typesetting, and review of the resulting proof before it is published in its final citable form. Please note that during the production process errors may be discovered which could affect the content, and all legal disclaimers that apply to the journal pertain. 


\section{INTRODUCTION}

The expansion of the neocortex in primate evolution may have been paralleled by increased innervation by dopamine (DA) (Gaspar et al., 1989; Lewis et al., 2001). In contrast to rodents, primates have denser and more extensive cortical dopaminergic (DAergic) innervation, with fibers invading all cortical layers in a regionally specific manner (Berger et al., 1991). However, data concerning variation in cortical DAergic afferents among primate species are lacking. Therefore, the aim of the current study was to conduct a comparative analysis of cortical DAergic innervation among humans, chimpanzees, and macaque monkeys.

The involvement of DA in cognitive processes that invoke the prefrontal cortex is welldocumented in humans and other species (Brozoski et al., 1979; Sawaguchi and GoldmanRakic, 1991; Kulisevsky 2000; Winterer and Weinberger, 2004). Functions that rely on DAergic input include working memory, language comprehension, reasoning, and overall intelligence (Boshes and Arbit, 1970; Sawaguchi and Goldman-Rakic, 1991; Arnsten et al., 1995; Goldman-Rakic, 1998; Dreher and Burnod, 2002; Nieoullon, 2002). There is also considerable evidence indicating that DA plays a significant role in a number of neuropsychiatric disorders presenting with cognitive deficits that appear to be exclusive to humans, including Alzheimer's disease, Parkinson's disease, and schizophrenia (Akil et al., 1999; Ciliax et al., 1999; Venator et al., 1999; Sutoo et al., 2001; Winterer and Weinberger, 2004). For these reasons, it has been proposed that the human neocortex might have an expanded and denser cortical DA innervation compared with other species (Previc, 1999), making it a candidate neural substrate for modification by natural selection in the evolution of human cognitive specializations.

In the present study, we performed a quantitative comparative analysis of tyrosine hydroxylase (TH)-immunoreactive axons in frontal cortical areas 4, 9, and 32 in humans, chimpanzees, and macaque monkeys. This study was designed to explore potential humanspecific adaptations of the cortical DAergic system that may contribute to cognitive specializations. For this evaluation, it was essential to also examine chimpanzees, as this species represents on of the closest living relatives of modern humans. In humans, prefrontal cortical areas 9 and 32 are involved in higher cognitive functions such as working memory and 'theory of mind' (TOM), respectively (Petrides et al., 1993; Petrides, 1995; Petrides, 2000; Johnson et al., 2002; Gallagher and Frith, 2003). As such, we hypothesized that differences in the DAergic innervation between humans and other primates would be found in these areas. For comparison, the primary motor cortex (area 4) was also examined. Species differences were not expected in area 4, as it is not associated with cognition and is thought to perform a similar function across primates (Rizzolatti et al., 1998; Kaas, 2004).

\section{EXPERIMENTAL PROCEDURES}

\section{Specimens}

The nonhuman brain specimens for this research included Moor macaques (Macaca maura, 4 females, 2 males, age range 5-10 years) and common chimpanzees (Pan troglodytes, 3 females, 3 males, age range 17-35 years). Human brain specimens were provided by Northwestern University Alzheimer's Disease Center Brain Bank (3 women, 3 men, age range 35-54 years). All human and nonhuman individuals were adult, non-geriatric, and free of gross neuropathologic abnormalities. The human cases showed no evidence of dementia before death and all individuals received a score of zero for the CERAD senile plaque grade (Mirra et al., 1999) and the Braak and Braak (1991) neurofibrillary tangle stage. The 
nonhuman subjects were housed in social groups. The age, sex, brain weight, and postmortem interval for each specimen can be found in Table 1 .

\section{Fixation}

The macaque monkeys were perfused transcardially with $4 \%$ paraformaldehyde as part of unrelated experiments following methods described previously (Hof and Nimchinsky, 1992; Hof et al., 1996). Chimpanzee and human brains were collected postmortem and fixed by immersion in $10 \%$ buffered formalin for 7-10 days, then transferred to a $0.1 \mathrm{M}$ phosphatebuffered saline (PBS, $\mathrm{pH} 7.4$ ) solution containing $0.01 \%$ sodium azide and stored at $4{ }^{\circ} \mathrm{C}$ to prevent further tissue shrinkage and blockade of antigens. The postmortem interval (PMI) prior to fixation for chimpanzee brains never exceeded 14 hours. For human cases, the PMI ranged from 6 to 17 hours.

\section{Sample processing}

All samples derived from the left hemisphere. For macaque and chimpanzee brains, the entire frontal lobe was removed just rostral to the primary motor cortex as a coronal slab, including areas 9 and 32. For macaque specimens, the occipital lobe was removed rostral to the lunate sulcus. This resulted in three "blocks" for each macaque left hemisphere, the middle block containing the primary motor cortex (area 4). The region of hand representation in the chimpanzee primary motor cortex had been dissected from the left hemisphere of each brain to be processed as small blocks as part of an unrelated project. This region was identified as the area on the lateral surface at the level of the middle genu located within the central sulcus (Yousry et al., 1997). Human samples were dissected from the regions of interest in $4 \mathrm{~cm}$-thick blocks by the donating brain bank. Prior to sectioning, samples were cryoprotected by immersion in a series of sucrose solutions $(10 \%, 20 \%$, and $30 \%)$.

Brain specimens were frozen on dry ice and cut to $40 \mu \mathrm{m}$-thick sections using a sliding microtome. As the brain samples were cut, sections were placed into individual microcentrifuge tubes containing freezer storage solution (30\% each distilled water, ethylene glycol, and glycerol and 10\% 0.244 M PBS) and numbered sequentially. Sections were stored at $-20^{\circ} \mathrm{C}$.

A 1-in-10 series for all samples was stained for Nissl substance with a solution of $0.5 \%$ cresyl violet to reveal cell somata. Nissl-stained sections were used to identify cytoarchitectural boundaries and to obtain neuron densities.

\section{Immunohistochemistry}

Floating tissue sections were stained using the avidin-biotin-peroxidase method. Sections were removed from the freezer and rinsed a minimum of $10 \times 5$ minutes in PBS. A 1-in-10 series (human samples and chimpanzee primary motor cortex) or a 1-in-20 series (macaque samples and chimpanzee frontal lobe) for each area was immunohistochemically stained for TH, to measure putative DA-containing fibers (Hof et al., 1995; Akil et al., 1999; Smiley et al., 1999) using a rabbit anti-TH polyclonal antibody (AB152, Chemicon, Temecula, CA). Sections were pretreated for antigen retrieval by incubating in $10 \mathrm{mM}$ sodium citrate buffer $(\mathrm{pH} 3.5)$ at $37^{\circ} \mathrm{C}$ for 30 minutes. Sections were then rinsed and endogenous peroxidase was quenched using a solution of $75 \%$ methanol, $2.5 \%$ hydrogen peroxide (30\%), and $22.5 \%$ distilled water for 20 minutes at room temperature. Sections were preblocked in a solution of PBS with 2\% normal goat serum and $0.3 \%$ Triton X-100 detergent Following this, sections were incubated in primary antibody diluted to 1:1,000 in PBS for 48 hours at $4^{\circ} \mathrm{C}$. After incubation in primary antibody, the tissue was incubated in biotinylated secondary antibody (1:200) in a solution of PBS and 2\% normal goat serum for 1 hour at room temperature. 
Sections were then incubated in avidin-peroxidase complex (PK-6100, Vector Laboratories, Burlingame, CA) for 1 hour at room temperature. A 3,3'-diaminobenzidine-peroxidase substrate with nickel solution enhancement was used as the chromogen (SK-4100, Vector Laboratories). Immunostained sections were counterstained with $0.5 \%$ methyl-green to visualize non-immunoreactive neurons and to aid in identifying layers within the cortex. Robust and full antibody penetration through the tissue sections was observed for each species, as demonstrated by axon staining through the $z$-axis (Figure 1). Negative controls omitted the primary antibody and omitted the secondary antibody. Omission of the primary or secondary antibody resulted in a complete absence of labeled axons.

Original photomicrographs were processed using Adobe Photoshop, v. 7.0 (San Jose, CA). Brightness, contrast, and sharpness were adjusted to obtain images that most closely resembled the appearance of histological features as seen through the microscope.

\section{Identifying cortical regions and layers}

Cortical regions of interest were identified based on topological location and distinctive regional cytoarchitecture recognizable on Nissl-stained sections. Cytoarchitectural features were relied upon for identification of cortical regions due to individual variation in their gross anatomical location (e.g., Amunts et al., 1996; Zilles et al., 1996; Petrides and Pandya, 1999; Rademacher et al., 2001). Cortical layers were analyzed separately as layers I, II, III, and V/VI. Because there is not a sharp border between the infragranular layers in all cortical areas examined, layers V and VI were analyzed together. Layer IV was not analyzed, as area 4 is agranular and area 32 is dysgranular. The borders between cortical areas tend not to be sharp or distinct, thus, sampling was limited to a representative region within the cortical areas of interest. That is, once the cortical area was identified in each series of Nissl-stained sections, the first and last sections that included the area were excluded from stereologic analyses to avoid sampling from transitional cortical regions. Further, due to the limited nature of human tissue availability, we were unable to sample uniformly through the entire extent of any of the cortical regions, and were thus limited to centrally located areas within the cortical regions of interest.

Area 9 is located in the dorsolateral prefrontal cortex, extending medially to the paracingulate sulcus of humans and the cingulate sulcus of macaque monkeys (Petrides and Pandya, 1999; Paxinos et al., 2000). This cortical area is expanded in anthropoids (i.e., monkeys, apes, and humans) with no obvious homologue in other mammals (Preuss and Goldman-Rakic, 1991; Aboitiz and Garcia, 1997). For this study, the part of area 9 sampled was located on the dorsal portion of the superior frontal gyrus in humans and chimpanzees, and corresponded to area 9L in macaque monkeys as designated by Paxinos et al. (2000) (Figure 2). Based on functional imaging studies of humans, this area has been shown to be involved in several cognitive processes, including inductive reasoning (Goel et al., 1997), TOM (Goel et al., 1995), and the retrieval phase of episodic and working memory (Marklund et al., 2007). Lesions restricted to this area impair working memory tasks that require ordering sample sets of 5 items or more in both humans and macaque monkeys, illustrating that this area is homologous in some of its functions among primates examined in the current study (Petrides et al., 1993; Petrides, 1995; Petrides, 2000).

Area 32 is defined as the portion of the paracingulate cortex anterior to the genu of the corpus callosum (Gallagher and Frith, 2003; Öngür et al., 2003) (see Figure 2). In humans, area 32 has been implicated in TOM, the ability to infer the mental states of others (Gallagher et al., 2000; Adolphs, 2001; Vogeley et al., 2001; Johnson et al., 2002; Gallagher and Frith, 2003). Macaques do not possess a strict homologue to the portion of area 32 that is activated in human TOM studies (i.e., anterior cingulate area 32) (Öngür et al., 2003). However, because we chose this area to investigate the evolution of this unique human 
behavioral capacity, for comparative purposes, we were limited to the most similar anatomical territory of the medial prefrontal cortex of macaques, defined as prelimbic cortex (i.e., prelimbic area 32) (Öngür et al., 2003). In chimpanzees, the cytoarchitecture of cortex within the anterior paracingulate gyrus was described by Bailey et al. (1950) closely to resemble area FDL in humans (von Economo and Koskinas, 1925), suggesting that they are homologous in structure.

\section{Axon length density}

Quantitative analyses were performed using computer assisted stereology. This system consisted of a Zeiss Axioplan 2 photomicroscope, equipped with an Optronics MicroFire camera, a Ludl XY motorized stage, Heidenhain $z$-axis encoder, and StereoInvestigator software, version 6 (MicroBrightField, Williston, VT). Once the cortical area of interest was identified in Nissl-stained sections, two to five equidistantly spaced sections per area of interest per individual were used. The variance in section number was dependent upon the number of sections available for that cortical area. The blocks of human tissue obtained from the brain bank were particularly thin and yielded only 20 to 30 sections in some instances. Once the area of interest was identified, the separate cortical layers (I, II, III, and V/VI) were individually traced using the software at low magnification (4x Zeiss Achroplan, N.A. 0.10). On the occasion when the methyl-green counterstain was too light to identify laminar boundaries, individual layers were traced from adjacent Nissl-stained sections and transferred to the immunostained sections.

Mean mounted section thickness was measured at every $5^{\text {th }}$ sampling location. Axon length was assessed using the SpaceBalls probe under Koehler illumination at 63x (Zeiss PlanAchromat, N.A. 1.4) (Calhoun and Mouton, 2000; Mouton et al., 2002; Calhoun et al., 2004; Kreczmanski et al., 2005). SpaceBalls is a stereological tool that places sampling hemispheres for lineal features in the context of a fractionator sampling scheme (Mouton, 2002). In this study, fibers were marked where they intersected the outline of a hemisphere of $10 \mu \mathrm{m}$ diameter in all samples. Total fiber length within the sampled volume of reference was calculated using the following equation (Calhoun et al., 2004):

$$
\mathrm{L}=2 \times(\mathrm{v} / \mathrm{a}) \times(\Sigma \text { is }) \times 1 / \text { asf } \times 1 / \text { ssf } \times 1 / \text { tsf }
$$

where v/a is the ratio of sampling frame volume to probe surface area, $\sum$ is is the sum of the number of intersections between fibers and sampling hemispheres, asf (area sampling fraction; the fraction of the total area sampled) is the area of the counting frame divided by the total area of the reference space, ssf (section sampling fraction) is the number of sections analyzed divided by the total number of sections through the reference space, and tsf (tissue sampling fraction) is the sampling box height divided by mean mounted section thickness. To obtain axon length density, the total fiber length was divided by the planimetric measurement of the reference volume that was sampled, as calculated by the StereoInvestigator software. Analyses of axon length densities were used to analyze speciesspecific cortical innervation patterns.

\section{Neuron density}

Neuron density was assessed using an optical disector combined with a fractionator sampling scheme. Layers II, III, and V/VI were outlined within the area of interest at low magnification (4x Zeiss Achroplan, N.A. 0.10). The optical disector probes were performed under Koehler illumination using a $63 x$ objective (Zeiss Plan-Apochromat, N.A. 1.4). Counting frames were set at $40 \times 40 \mu \mathrm{m}$. Neurons were counted when the nucleolus was in focus within the counting frame. Neurons were identified based on the presence of a large, 
lightly stained nucleus, a distinct nucleolus, and lightly stained proximal portions of dendritic processes (e.g., Sherwood et al., 2005). The counting frame height was set at $7 \mu \mathrm{m}$ to allow a guard zone of at least $2 \mu \mathrm{m}$ at the top and bottom of the sections. Neuron density was calculated as the sum of neurons counted with the optical disectors divided by the product of the disectors and the volume of the disector (Sherwood et al., 2005). To correct for tissue shrinkage in the $z$ axis, the height of the disector was multiplied by the ratio of the sectioned thickness $(40 \mu \mathrm{m})$ to the actual number weighted mean thickness (calculated from the section thickness that was empirically measured at every $5^{\text {th }}$ sampling location) after mounting and dehydration. No correction was necessary for the $x$ and $y$ dimensions because shrinkage in section surface area is minimal (Dorph-Petersen et al., 2001).

\section{Axon length density/neuron density ratio (ALv/Nv)}

The ratio of axon length density to neuron density (ALv/Nv) was used for comparative analyses among species rather than total axon length to avoid several confounding factors. First, cell density per unit volume can vary with changes in brain size (Haug, 1987; Sherwood et al., 2007). Second, PMI, method of fixation, and amount of time in fixative are factors that contribute to preprocessing tissue shrinkage. Additional tissue shrinkage may occur with histological and immunohistochemical procedures. Thus, the ratio of ALv/Nv allows for the evaluation of fiber density in the context of species differences in neuron density and also acts to standardize data for differential tissue shrinkage among species as well as between individuals.

\section{Statistical analyses}

Factorial analysis of variance (ANOVA) with repeated measures design was used to examine differences among macaques, chimpanzees and humans. The variables were THimmunoreactive (TH-ir) ALv/Nv for layers II, III, and V/VI. A $3 \times 3 \times 3$ mixed-model ANOVA was performed with cortical area $(9,32$, and 4$)$ and layer (II, III, and V/VI) as within-subjects measures and species as the between-subjects measure. Tukey's Honestly Significant Difference (HSD) post hoc tests were used to analyze significant results indicated by the ANOVA analyses. Separate analyses were conducted for TH-ir axon length density to examine innervation patterns independent of neuron densities and species effects. For axon length density, a $3 \times 4$ (area $\times$ layer) repeated measures ANOVA was used to analyze the pattern differences between areas and layers within each species. Tukey's HSD post hoc tests were used to evaluate significant results.

To assess whether PMI affected the intensity of immunohistochemical staining, nonparametric Spearman's correlation coefficients were calculated for PMI and TH-ir ALv/ $\mathrm{Nv}$ in humans. Data on specific PMI were not available for the chimpanzee sample, and PMI was not applicable for the macaques as they were perfused.

Even though our sample was restricted to non-geriatric individuals in order to control for the potentially confounding factor of age-related declines in cortical neuromodulator density, we used Spearman's rank order correlation to empirically test whether there was a relationship between age and TH-ir ALv/Nv within each species. $\alpha$ was set at 0.05 for all statistical tests.

\section{Methodological concerns}

Tyrosine hydroxylase (TH) is the rate-limiting enzyme for DA synthesis, as well as for the synthesis of norepinepherine and epinephrine (Cooper et al., 2002). In some species, such as the rabbit, TH-ir axons can be primarily noradrenergic (Wang et al., 1996). However, several studies in primate species have demonstrated that TH and dopamine $\beta$-hydroxylase, the enzyme required for norepinepherine synthesis, are not extensively colocalized in immunoreactive axons ( Akil and Lewis, 1993; Gaspar et al., 1989; Lewis et al., 1987; 
Melchitzsky and Lewis, 2000). This indicates that TH is predominantly localized in DAergic axons within the primate cortex, and as such, TH may be considered a reliable marker for DAergic projections and neurons in primates. It should be noted, however, that we did not perform colocalization analyses for the TH antibody used in the present study.

Another methodological concern pertains to the effect of immersion (humans and chimpanzees) versus perfusion (macaques) methods of fixation on the reliability of immunohistochemistry. Perfusion is the most effective method of preservation for immunohistochemical procedures (Evers and Uylings, 1997; Evers et al., 1998; Shiurba et al., 1998; Jiao et al., 1999). If this were a factor in this study, it would be expected that axons would be overrepresented in all layers and areas of the macaques. However, staining was robust in all species and such an overrepresentation in macaques was not observed. This is evident in the results, wherein macaques do not exhibit uniformly greater densities than either humans or chimpanzees. Rather, the amount of variation observed in macaques relative to other species is layer- and area-specific and not in one consistent direction.

\section{RESULTS}

TH-ir axons were present in all cortical layers of the areas examined in the three species. Figures 3, 4, and 5 show tracings of TH-ir axons in each area for each species and examples of staining are shown in Figures 6, 7, 8 and 9. There was considerable variation among individuals, but this variation was not consistently correlated with PMI among humans. Of the possible 9 correlations ( 3 layers $\times 3$ cortical areas) with PMI in humans, 8 were not statistically significant. Only the correlation between PMI and TH Alv/Nv in layers V/VI of area 9 was significant (Spearman's rho $=-0.81, \mathrm{p}<0.05$ ). Also, there was no association between $\mathrm{TH} A L v / \mathrm{Nv}$ and age within any of the three species, i.e., the oldest individuals per species did not have lower values than younger individuals. Descriptions of TH-ir fiber distributions in the cerebral cortex have been reported for humans (Gaspar et al., 1989; Benavides-Piccione and DeFelipe, 2003) and long-tailed macaques (Macaca fascicularis) (Lewis et al., 1987). The present findings are in accordance with these earlier reports.

\section{Qualitative description}

The morphology of TH-labeled fibers consisted of straight, smooth axons and varicose fibers. Consistent with prior descriptions, the varicose fibers observed could be categorized into two major morphological groups: 1) curvilinear or rectilinear fibers with regularly spaced varicosities and 2) fine axons with irregularly spaced oblong swellings (Gaspar et al., 1989; Benavides-Piccione et al., 2005). Of the three cortical areas examined, area 4 demonstrated the densest innervation in all species. Qualitatively, area 32 appeared to have fewer TH-ir fibers than area 9 in macaques, but this difference was not notable in either chimpanzees or humans.

Layer I was densely innervated and contained mostly horizontally projecting fibers in all cortical areas in all three species. However, while macaques and chimpanzees exhibited THir fibers throughout layer I in the areas examined, humans had relatively sparse innervation at the top of layer I, and fibers were much denser in the lower portion for areas 9 and 32. In human area 4, TH-ir fibers were dense throughout layer I. This sublaminar pattern of layer I TH-ir axon distribution in humans was also noted by Gaspar et al. (1989). In layers II-VI of all species, fibers did not have a preferred direction of orientation and formed dense networks of thin, varicose axons. In chimpanzees, layer III of areas 9, 32, and 4 appeared to have considerably more TH-ir fibers than layer III of macaques or humans. However, this distinction was less evident when comparing area 4 between species. Qualitatively, layers II and V/VI appeared more or less comparable among species. TH-ir fibers were noted in the 
white matter immediately subjacent to layer VI in all species. However, these fibers were more abundant in macaques, particularly in area 32.

The presence of "coils" was noted in all areas of humans and chimpanzees (Figure 10). Coils are dense clusters formed by intertwined TH-ir varicose fibers, and have been described in humans (Gaspar et al., 1989; Benavides-Piccione and DeFelipe, 2003). Coils were most commonly observed in layer III, although they occurred in all layers of both species. Although intertwined TH-ir fibers were observed in macaques, particularly in area 4 , no structures directly comparable to the dense coils that were observed in humans and chimpanzees were found.

To determine if TH-ir axon coils were an artifact of fixation (i.e., chimpanzee and human brains were immersion fixed and macaque brains were perfused), we immunostained sections through areas 9 and 32 from 18 additional individuals representing six Old World monkey species (golden monkey, patas monkey, black and white colobus, Francois's langur, olive baboon, and golden mangabey) and three great ape species (bonobo, gorilla, and orangutan). Coils of TH-ir axons were noted within the cortex of each of the great apes, but were absent in all of the Old World monkey species.

\section{Within-species analyses}

For neuron density counts, an average of $102.4 \pm 22.5$ (mean \pm standard deviation) sampling sites was placed in each layer/individual/cortical area, with a total of 16,591 sampling sites investigated and 40,201 neurons counted. The mean coefficient of error related to sampling (CE, Schmitz and Hof, 2000) was 0.06 with a standard deviation of 0.02 .

For TH-ir axon length estimation, an average of $99.4 \pm 24.3$ sampling hemispheres was placed in each layer/individual/cortical area. A total of 21,462 sampling hemispheres were used, with 80,291 intersections counted.

Table 2 lists the mean TH-ir axon length density for each species/layer/area. The $4 \times 3$ repeated-measures ANOVA used for the analysis of TH-ir axon length density within macaques showed a significant interaction between layer and area $\left(\mathrm{F}_{6,30}=3.3, \mathrm{p}<0.05\right.$; Figure 11a), and significant main effects of layer $\left(\mathrm{F}_{3,15}=140.6, \mathrm{p}<0.05\right)$ and area $\left(\mathrm{F}_{2,10}=\right.$ $10.6, \mathrm{p}<0.05)$. The results of the chimpanzee analysis yielded a significant interaction $\left(\mathrm{F}_{6,30}=3.1, \mathrm{p}<0.05\right.$; Figure $\left.11 \mathrm{~b}\right)$, and a significant main effect of layer $\left(\mathrm{F}_{3,15}=14.7, \mathrm{p}<\right.$ $0.05)$. The main effect of area was not significant $\left(\mathrm{F}_{2,10}=2.5, \mathrm{p}>0.05\right)$. In humans, the interaction was significant $\left(\mathrm{F}_{6,30}=5.9, \mathrm{p}<0.05\right.$; Figure 11c), as was the main effect of layer $\left(F_{3,15}=29.4, p<0.05\right)$. The main effect of area was not significant $\left(F_{2,10}=2.2, p>0.05\right)$. Post hoc Tukey HSD tests were used to evaluate the significant interactions for each species. Comparisons were made between layers within each cortical area (Table 3). Differences of layers between cortical regions are also reported (Table 4). The results demonstrate significant differences in the patterns of dopaminergic axon length density among humans, chimpanzees, and macaques, independent of neuron densities.

In macaques, layers I and II received the densest contingent of TH-ir axons in all three cortical areas, with layer I having a higher axon length density than any other layer, and layer II having a greater density than either layer III or V/VI (see Table 3). Layers III and V/ VI were equally innervated for each area. In the comparisons between cortical areas (see Table 4), area 4 displayed a higher axon length density in all layers relative to area 32, and had a greater density only in layer III relative to area 9. Additionally, area 9 exhibited a higher density in layers I and II when compared to the same layers of area 32. 
The analysis of chimpanzee data revealed a different pattern. Layer I was more densely innervated than layer II only in areas 9 and 4 (see Table 3). Further, layer I had a higher density relative to layer III only in area 4 , and was greater relative to layers V/VI in each of the three cortical areas. Only one difference was detected in comparisons made between cortical areas; axon length density in layer I of area 4 was significantly higher than layer I of area 32 (see Table 4). No other differences were detected.

Humans were different from both macaques and chimpanzees. Layers V/VI had a significantly higher density than layers II and III in both areas 9 and 32, and layer I of area 9 (see Table 3). Layer I was more densely innervated than layer II in area 4 and layer III in areas 4 and 32. Among cortical areas, area 32 had denser innervation in layer I relative to that of area 9 (see Table 4). Area 32 also had a higher density in layer V/VI relative to area 4. Finally, layer I displayed a greater density in area 4 relative to area 9.

\section{Among-species analyses}

The mean $\mathrm{ALv} / \mathrm{Nv}$ and standard deviation for each layer and cortical area for macaques, chimpanzees, and humans are shown in Table 5 . In the $3 \times 3 \times 3$ ANOVA with repeatedmeasures design, the three-way interaction, layer $\times$ area $\times$ species, was statistically significant $\left(\mathrm{F}_{8,60}=2.2, \mathrm{p}<0.05\right)$. Corresponding two-way interaction terms were significant as well: layer $\times$ species $\left(\mathrm{F}_{4,30}=42.9, \mathrm{p}<0.05\right)$; area $\times$ species $\left(\mathrm{F}_{4,30}=3.3, \mathrm{p}<\right.$ $0.05)$; layer $\times$ area $\left(\mathrm{F}_{4}, 60=3.3, \mathrm{p}<0.05\right)$. Finally, all main effects were statistically significant: layer $\left(\mathrm{F}_{2,30}=53.1, \mathrm{p}<0.05\right)$; area $\left(\mathrm{F}_{2,30}=4.4, \mathrm{p}<0.05\right)$; and species $\left(\mathrm{F}_{2,15}=\right.$ $8.6, \mathrm{p}<0.05)$. The interaction among layer, area, and species is graphically represented in Figure 12.

Post hoc Tukey HSD tests of the three-way interaction revealed no differences between species. Comparisons within each species between cortical areas were also made (Table 6). In humans, none of the layers were different between area 4 and area 9, nor were there differences between area 4 and area 32 . The same pattern held for chimpanzees, with no laminar differences detected between area 4 and area 9, or between area 4 and area 32. For macaques, there were no differences in layer II between area 4 and area 9 , or between area 4 and area 32. Layer III and layers V/VI were significantly different between area 4 and area 9 as well as between area 4 and area 32 for macaques, with area 4 having denser ALv/Nv. No differences were detected within any of the species between areas 9 and 32 .

\section{DISCUSSION}

This is the first rigorous analysis of cortical DAergic innervation between human and nonhuman primate species and the first characterization of cortical DAergic innervation in the chimpanzee. Direct comparative studies of the DAergic system have heretofore been restricted to analyses of rats versus humans and macaque monkeys. The differences between these two groups in both the organization of the frontal cortex and in DAergic innervation of this area are striking and suggest evolutionary changes that paralleled increases in both the size and functional differentiation of the cerebral cortex (e.g., van Eden et al., 1987; Berger et al., 1991; Preuss, 1995; Sesack et al., 1995; Williams and Goldman-Rakic, 1998). For example, the primary motor cortex is thought to be the latest development of a primary cortical field in mammalian neocortical evolution (Sanides, 1970; Kaas, 2004), and is the most densely DA-innervated cortical area in primates (Lewis et al., 1987; Gaspar et al., 1989; Berger et al., 1991). However, homologous motor areas in rodents are sparsely innervated (Berger et al., 1991). Also, the molecular layer is a widespread target for dense DAergic innervation in all areas of the macaque and human neocortex, but DAergic innervation of layer I is restricted to only a few areas in rats. Indeed, the motor, parietal, and temporal cortical areas of rats do not have DAergic afferents in the molecular layer. Finally, 
primates share neurochemical properties of DAergic afferents that are not found in rats, suggesting that the DAergic neurons innervating the frontal cortex of humans and nonhuman primates are fundamentally distinct from the DAergic neurons that innervate the infragranular layers in rodents (Studler et al., 1988; Gaspar et al., 1990; Berger et al., 1991; Berger et al., 1992). Such significant differences between rodents and primates prevent direct comparisons of cortical DAergic function within the frontal cortex. The importance of these differences in the presynaptic component of the cortical DAergic system between these taxonomic groups is further supported by the finding that primates have an accelerated rate of protein evolution for the DA receptor gene, DRD2, relative to rodents (Dorus et al., 2004).

A broader view of phylogenetic differences has been provided by Hof and collaborators in an analysis of cortical TH-ir axon distribution in the harbor porpoise and pilot whale (Hof et al., 1995). Their findings revealed a different pattern of innervation of auditory and visual cortices of cetaceans compared to that of other mammals. Most other mammals share in common a sparser DAergic innervation in primary sensory cortices relative to all other cortical areas. This is particularly true of the primary visual cortex, where humans and other primates exhibit TH-ir axons only in layer I and DAergic axons are rarely found in rodent visual cortex (Gaspar et al., 1989; Berger et al., 1991). In contrast, the cetacean primary visual cortex is innervated throughout all layers, and it is more densely innervated than the auditory cortex, whereas the reverse is true for the other mammals (Hof et al., 1995). Such phylogenetic differences strongly suggest a potential role for this neurotransmitter in brain evolution.

Additional lines of evidence suggest that DAergic systems may have been subtly altered in human evolution. For example, there is considerable evidence indicating that DA dysfunction plays an important role in a number of neuropsychiatric disorders presenting with cognitive deficits that preferentially afflict humans, including Alzheimer's disease, Parkinson's disease, and schizophrenia (Akil et al., 1999; Ciliax et al., 1999; Venator et al., 1999; Sutoo et al., 2001; Winterer and Weinberger, 2004). Further, a substantial decrease in TH-ir axons in layer VI of area 9 occurs in schizophrenic subjects (Akil et al., 1999), potentially underlying working memory deficits in this disease (Akil et al., 1999; AbiDargham, 2004). Humans appear to be uniquely susceptible to these disease states that are associated with devastating cognitive deficits. This susceptibility may be due to an increased reliance on DAergic systems to support intellectual capabilities. Based on this evidence, we hypothesized that humans would have significantly more cortical TH-ir fibers, particularly in prefrontal cortical areas relative to other primate species. Previc (1999) proposed that DAergic systems increased and expanded within the human neocortex, and that this increase was responsible for the origins of human intelligence. However, the results of the present study do not fully support this hypothesis and indicate that humans do not exhibit an overt increase of DAergic innervation within the cerebral cortex in comparison to their close phylogenetic relatives, chimpanzees.

Nonetheless, the evaluation of TH-ir axon length density within each species demonstrated interesting differences among humans, chimpanzees, and macaques, which might have functional implications. Although these differences cannot be used in direct between-species comparisons because they do not take into account differential tissue shrinkage, these analyses illustrate that the species examined vary in regional pattern of cortical DAergic innervation within the frontal cortex (see Tables 3 and 4). Briefly, macaques display a denser innervation in layers I and II relative to layers III and V/VI in all three cortical regions examined. In contrast, humans display a much higher innervation in layers V/VI only in areas 9 and 32. The pattern of innervation for chimpanzees is altogether different, with layer I being the most densely innervated layer only in area 4. 
Direct comparisons of ALv/Nv in each layer and area among humans, chimpanzees, and macaques found general similarities in laminar patterns, however, comparisons made between the cortical areas involved in cognition versus the primary motor cortex revealed interesting species differences. Of the different cortical regions, the primary motor cortex has the densest DAergic innervation in primates, including humans (Lewis et al., 1987; Gaspar et al., 1989). Our analyses detected significant differences in layers III and V/VI of macaques. Density of TH-ir axon length relative to neuron density was significantly less in these layers in areas 9 and 32 when compared to area 4. No such differences were found in either humans or chimpanzees, with areas 9 and 32 being as densely innervated as area 4 . These results suggest an evolutionary shift towards relatively denser DAergic innervation of layers III and V/VI of these prefrontal areas in humans and chimpanzees.

The morphologic appearance of TH-ir fibers in the molecular layer of areas 9 and 32 in humans was considerably different from the distribution observed in macaques and chimpanzees. Innervation of layer I in humans is mostly restricted to the lower portion of the layer, whereas the entire layer is equally innervated in the other species. This observation was previously made in humans, with the suggestion that this pattern could have evolutionary implications (Gaspar et al., 1989). This pattern of sublaminar innervation in the molecular layer has been reported in agranular cortices of long-tailed macaque monkeys (Berger et al., 1988), but may have been extended in humans to include both agranular and granular cortices (Gaspar et al., 1989).

Another species difference concerns the coil-like accumulations of TH-ir fibers within the cortical mantle of only humans and chimpanzees, most commonly observed in layer III. Although coils of TH-ir axons were previously described in humans, the functional significance of these structures is unknown (Gaspar et al., 1989; Benavides-Piccione and DeFelipe, 2003). We did not detect the presence of coils in any of the areas examined in macaques, consistent with earlier reports. A detailed analysis that included long-tailed macaques (Macaca fascicularis) and squirrel monkeys (Saimiri sciureus) did not report the presence of TH-ir coils within the cortical mantle (Lewis et al., 1987). Another study of Macaca fascicularis reported "clusters" of DAergic fibers in the upper portion of layer III of the motor areas 4 and 6, but not in other cortical areas (Berger et al., 1988). Similarly, we did not find any coil-like TH-stained structures in the frontal cortex of any species of Old World monkeys that we examined, whereas coils were consistently present in great apes. These results suggest that TH-ir axon coils represent an important morphological variant that is present only in apes and humans.

Interestingly, analogous morphological features (i.e., coils/clusters of axons) have been reported for cholinergic and serotonergic axons in human and chimpanzee cortex, and have been interpreted to represent local events of cortical plasticity or circuit changes (Mesulam et al., 1992; Raghanti et al., 2007; Raghanti et al., 2008). Neuromodulatory transmitters have well described functions in modifying cortical neuron response properties as mediated by numerous receptor subtypes (Gu, 2002; von Bohlen und Halbach and Dermietzel, 2006). Specific effects include long-term potentiation and long-term inhibition, depending on the properties of the post-synaptic element. The finding that coils of TH-ir axons are most numerous in layer III is important because of its putative role as the terminal input layer in corticocortical connections (e.g., Fuster, 1997). If coils are indicative of cortical plasticity, these findings suggest increased synaptic reorganization that may be manifested in increased cognitive and behavioral flexibility. Specifically, capacities such as an awareness of "self" (Gallup, 1982), transmission of social traditions (Whiten and van Schaik, 2001; van Schaik and Pradhan, 2003), and symbolic language acquisition (Gardner and Gardner, 1985) that are unique to the great ape and human clade may require a greater capacity for cortical plasticity. 
There exist limitations for interpreting results from comparative studies that are noteworthy. First, there is little functional evidence indicating that other species possess strict homologues for human cortical areas. Although this limits interpretation of comparative results to some extent, it does allow for an evaluation of potential human-specific attributes. This is particularly relevant for our analysis of area 32 . The integrity of this cortical area is necessary for the putatively unique human capacity of TOM (e.g., Gallagher and Frith, 2003). Because macaques do not possess a structural homologue to human area 32 , it was necessary to analyze the closest anatomical region to evaluate the emergence of potential neuroanatomical substrates that support TOM in humans. In addition, while the current study detected differences between the prefrontal areas versus primary motor cortex, further cortical regions need be examined to determine if this is a functional adaptation, or a general feature of all prefrontal cortical areas on a phylogenetic level.

Currently, very little is known regarding differences in cortical histology between humans other species (Preuss, 2000; Preuss, 2006; Sherwood and Hof, 2007). This report represents a first step towards a broader understanding of human-specific cortical DAergic specializations that may support the evolution of cognition. Future studies that include a wider variety of primate species, cortical areas, and incorporate additional measures of innervation (such as varicosity densities) will further elucidate the functional roles of DA within the cortical mantle.

\section{Conclusions}

Several differences in cortical DAergic innervation were observed among species which may have functional implications. Specifically, humans exhibited a sublaminar pattern of innervation in layer I of areas 9 and 32 that differed from macaques and chimpanzees. In addition, in statistical analyses of axon length density within species, humans displayed a greater density of innervation to infragranular layers exclusively in cortical areas involved in high-level cognitive processing (areas 9 and 32), but not in primary motor cortex. The other species displayed different regional and laminar variation in DAergic innervation. Macaques consistently displayed denser innervation of layers I and II relative to layers III and V/VI for all cortical areas examined. In contrast, chimpanzees did not demonstrate a consistent direction of denser innervation. Further, the among-species analysis of ALv/Nv revealed that humans and chimpanzees together deviated from macaques in having increased DAergic afferents in layers III and V/VI of areas 9 and 32. Finally, morphological specializations that may be indicative of cortical plasticity events were observed in humans and chimpanzees, but not macaques.

Taken together, these findings suggest that significant modifications of DA's role in cortical organization occurred in the evolution of the apes, with further changes in the descent of humans. However, we did not find an overt quantitative increase in cortical DAergic innervation in humans relative to chimpanzees. In this regard, our results highlight the importance of including chimpanzees in comparative neuroanatomical studies to determine human brain specializations (e.g., Preuss, 2000). The addition of chimpanzees in this analysis has enhanced our ability to understand the role of cortical DAergic innervation in humans in a phylogenetic perspective. Finally, the distinctive innervation patterns and morphological specializations shared by humans and chimpanzees imply functional specializations of cortical DAergic innervation that may provide new insight into normal and pathological functioning of the human brain.

\section{Acknowledgments}

This work was supported by the National Science Foundation (BCS-0515484 and BCS-0549117), National Institutes of Health (NS42867), the Wenner-Gren Foundation for Anthropological Research, and the James S. 
McDonnell Foundation (22002078). Brain material used in this study was loaned by the Great Ape Aging Project (USPHS/NIH grant AG14308, “A Comparative Neurobiology of Aging Resource," J. Erwin, PI), the Foundation for Comparative and Conservation Biology, and the Northwestern University Alzheimer's Disease Center Brain Bank (NADC grant P30 AG13854).

\section{Abbreviations}

$\begin{array}{ll}\text { ALv } & \text { axon length density } \\ \text { ALv/Nv } & \text { the ratio of axon length density to neuron density } \\ \text { DA } & \text { dopamine } \\ \text { DAergic } & \text { dopaminergic } \\ \text { PBS } & \text { phosphate-buffered saline } \\ \text { PMI } & \text { postmortem interval } \\ \text { TH } & \text { tyrosine hydroxylase } \\ \text { TH-ir } & \text { tyrosine hydroxylase-immunoreactive } \\ \text { TOM } & \text { theory of mind }\end{array}$

\section{References}

Abi-Dargham A. Do we still believe in the dopamine hypothesis? New data bring new evidence. Int J Neuropsychopharmacol. 2004; 7:S1-S5. [PubMed: 14972078]

Akil M, Lewis DA. The dopaminergic innervation of monkey entorhinal cortex. Cereb Cortex. 1993; 3:533-550. [PubMed: 7907902]

Akil M, Pierri JN, Whitehead RE, Edgar CL, Mohila C, Sampson AR, Lewis DA. Lamina-specific alterations in the dopamine innervation of the prefrontal cortex in schizophrenic subjects. Am J Psychiatry. 1999; 156:1580-1589. [PubMed: 10518170]

Amunts K, Schlaug G, Schleicher A, Steinmetz H, Dabringhaus A, Roland PE, Zilles K. Asymmetry in the human motor cortex and handedness. Neuroimage. 1996; 4:216-222. [PubMed: 9345512]

Arnsten A, Cai J, Steere J, Goldman-Rakic PS. Dopamine D2 receptor mechanisms contribute to agerelated cognitive decline: the effects of quinpirole on memory and motor performance in monkeys. $J$ Neurosci. 1995; 15:3429-3439. [PubMed: 7751922]

Bailey, P.; von Bonin, G.; McCulloch, WS. The Isocortex of the Chimpanzee. University of Illinois Press; Urbana, IL: 1950.

Benavides-Piccione R, Arellano JI, DeFelipe J. Catecholaminergic innervation of pyramidal neurons in the human temporal cortex. Cereb Cortex, Advance Access. 2005:1-8.

Benavides-Piccione R, DeFelipe J. Different populations of tyrosine-hydroxylase-immunoreactive neurons defined by differential expression of nitric oxide synthase in the human temporal cortex. Cereb Cortex. 2003; 13:297-307. [PubMed: 12571119]

Berger B, Gaspar P, Verney C. Dopaminergic innervation of the cerebral cortex: unexpected differences between rodents and primates. Trends Neurosci. 1991; 14:21-27. [PubMed: 1709528]

Berger B, Gaspar P, Verney C. Colocalization of neurotensin in the mesocortical dopaminergic system. Restricted regional and laminar distribution in rat, lack of colocalization in human. Ann N Y Acad Sci. 1992; 668:307-310. [PubMed: 1361118]

Berger B, Trottier S, Verney C, Gaspar P, Alvarez C. Regional and laminar distribution of the dopamine and serotonin innervation in the macaque cerebral cortex: A radioautographic study. $\mathrm{J}$ Comp Neurol. 1988; 273:99-119. [PubMed: 3209731]

Boshes B, Arbit J. A controlled study of the effect of L-DOPA upon selected cognition and behavioral functions. Trans Am Neurol Assoc. 1970; 95:59-63. [PubMed: 4936414]

Braak H, Braak E. Neuropathological staging of Alzheimer-related changes. Acta Neuropathol. 1991; 82:239-259. [PubMed: 1759558] 
Brozoski TJ, Brown RM, Rosvold HE, Goldman PS. Cognitive deficit caused by regional depletion of dopamine in prefrontal cortex of rhesus monkey. Science. 1979; 205:929-932. [PubMed: 112679]

Calhoun ME, Mao Y, Roberts JA, Rapp PR. Reduction in hippocampal cholinergic innervation is unrelated to recognition memory impairment in aged rhesus monkeys. J Comp Neurol. 2004; 475:238-246. [PubMed: 15211464]

Calhoun ME, Mouton PR. Length measurement: new developments in neurostereology and 3D imagery. J Chem Neuroanat. 2000; 20:61-69. [PubMed: 11074344]

Ciliax BJ, Drash GW, Staley JK, Haber S, Mobley CJ, Miller GW, Mufson EJ, Mash DC, Levey AI. Immunocytochemical localization of the dopamine transporter in human brain. The J Comp Neurol. 1999; 409:38-56.

Cooper, J.; Bloom, FE.; Roth, R. The Biochemical Basis of Neuropharmacology. Oxford University Press; New York: 2002.

Dorph-Petersen KA, Nyengaard JR, Gundersen HJ. Tissue shrinkage and unbiased stereological estimation of particle number and size. J Microsc. 2001; 204:232-246. [PubMed: 11903800]

Dorus S, Vallender EJ, Evans PD, Anderson JR, Gilbert SL, Mahowald M, Wyckoff GJ, Malcom CM, Lahn BT. Accelerated evolution of nervous system genes in the origin of Homo sapiens. Cell. 2004; 119:1027-1040. [PubMed: 15620360]

Dreher J, Burnod Y. An integrative theory of the phasic and tonic modes of dopamine modulation in the prefrontal cortex. Neural Netw. 2002; 15:583-602. [PubMed: 12371514]

Evers P, Uylings HBM. An optimal antigen retrieval method suitable for different antibodies on human brain tissue stored for several years in formaldehyde fixative. J Neurosci Methods. 1997; 72:197-207. [PubMed: 9133585]

Evers P, Uylings HBM, Suurmeijer AJH. Antigen retrieval in formaldehyde-fixed human brain tissue. Methods. 1998; 15:133-140. [PubMed: 9654460]

Fuster, JM. The Prefrontal Cortex: Anatomy, Physiology, and Neuropsychology of the Frontal Lobe. ed Third. Lippincottt-Raven Publishers; Philadelphia: 1997.

Gallagher HL, Frith CD. Functional imaging of 'theory of mind'. Trends in Cog Neurosci. 2003; 7:7783.

Gallup GG Jr. Self-awareness and the emergence of mind in primates. Am J Primatol. 1982; 2:237248.

Gardner B, Gardner RA. Signs of intelligence in cross-fostered chimpanzees. Philos Trans R Soc Lond Biol Sci. 1985; 308:159-176. [PubMed: 2858873]

Gaspar P, Berger B, Febvret A. Neurotensin innervation of the human cerebral cortex: lack of colocalization with catecholamines. Brain Res. 1990; 530:181-195. [PubMed: 2265353]

Gaspar P, Berger B, Febvret A, Vigny A, Henry JP. Catecholamine innervation of the human cerebral cortex as revealed by comparative immunohistochemistry of tyrosine hydroxylase and dopaminebeta-hydroxylase. J Comp Neurol. 1989; 279:249-271. [PubMed: 2563268]

Goldman-Rakic PS. The cortical dopamine system: Role in memory and cognition. Adv Pharmacol. 1998; 42:707-711. [PubMed: 9327997]

$\mathrm{Gu}$ Q. Neuromodulatory transmitter systems in the cortex and their role in cortical plasticity. Neuroscience. 2002; 111:815-835. [PubMed: 12031406]

Haug H. Brain sizes, surfaces, and neuronal sizes of the cortex cerebri: a stereological investigation of man and his variability and a comparison with some mammals (primates, whales, marsupials, insectivores, and one elephant). Am J Anat. 1987; 180:126-142. [PubMed: 3673918]

Hof PR, Glezer II, Revishchin AV, Bouras C, Charnay Y, Morgne PJ. Distribution of dopaminergic fibers and neurons in visual and auditory cortices of the harbor porpoise and pilot whale. Brain Res Bull. 1995; 36:275-284. [PubMed: 7697381]

Hof PR, Nimchinsky EA. Regional distribution of neurofilament and calcium-binding proteins in the cingulate cortex of the macaque monkey. Cereb Cortex. 1992; 2:456-467. [PubMed: 1282402]

Hof PR, Ungerleider LG, Webster MJ, Gattass R, Adams MM, Sailstad CA, Morrison JH. Neurofilament protein is differentially distributed in subpopulations of corticortical projectiion neurons in the macaque monkey visual pathways. J Comp Neurol. 1996; 376:112-127. [PubMed: 8946287] 
Jiao Y, Sun Z, Lee T, Fusco FR, Kimble TD, Meade CA, Cuthbertson S, Reiner A. A simple and sensitive antigen retrieval method for free-floating and slide-mounted tissue sections. J Neurosc Methods. 1999; 93:149-162.

Johnson SC, Baxter LC, Wilder LS, Pipe JG, Heiserman JE, Prigatano GP. Neural correlates of selfreflection. Brain. 2002; 125:1808-1814. [PubMed: 12135971]

Kaas JH. Evolution of somatosensory and motor cortex in primates. The Anat Rec Part A. 2004; 281A: 1148-1156.

Kreczmanski P, Schmidt-Kastner R, Heinsen H, Steinbusch HWM, Hof PR, Schmitz C. Stereological studies of capillary length density in the frontal cortex of schizophrenics. Acta Neuropathol. 2005; 109:510-518. [PubMed: 15886994]

Kulisevsky J. Role of dopamine in learning and memory: Implications for the treatment of cognitive dysfunction in patients with Parkinson's disease. Drugs Aging. 2000; 16:365-379. [PubMed: 10917074]

Lewis DA, Campbell MJ, Foote SL, Goldstein M, Morrison JH. The distribution of tyrosine hydroxylase-immunoreactive fibers in primate neocortex is widespread but regionally specific. $\mathrm{J}$ Neurosci. 1987; 7:279-290. [PubMed: 2879896]

Lewis DA, Melchitzky DS, Sesack SR, Whitehead RE, Auh S, Sampson A. Dopamine transporter immunoreactivity in monkey cerebral cortex: regional, laminar, and ultrastructural localization. J Comp Neurol. 2001; 432:119-136. [PubMed: 11241381]

Melchitzky DS, Lewis DA. Tyrosine hydroxylase and dopamine transporter-immunoreactive axons in the primate cerebellum. Neuropsychopharacol. 2000; 22:466-472.

Mesulam MM, Hersh LB, Mash DC, Geula C. Differential cholinergic innervation within functional subdivisions of the human cerebral cortex: a choline acetyltransferase study. J Comp Neurol. 1992; 318:316-328. [PubMed: 1374768]

Mirra SS, Heyman A, McKeel S, Sumi SM, Crain BJ, Brownlee LM, Vogel FS, Hughes JP, van Belle G, Berg L. The consortium to establish a registry for Alzheimer's disease (CERAD). Neurology. 1991; 41:479-486. [PubMed: 2011243]

Mouton, PR. Principles and Practices of Unbiased Stereology: An Introduction for Bioscientists. The Johns Hopkins University Press; Baltimore and London: 2002.

Mouton PR, Gokhale AM, Ward NL, West MJ. Stereological length estimation using spherical probes. J Microsc. 2002; 206:54-64. [PubMed: 12000563]

Nieoullon A. Dopamine and the regulation of cognition and attention. Prog Neurobiol. 2002; 67:5383. [PubMed: 12126656]

Öngür D, Ferry AT, Price JL. Architectonic subdivision of the human orbital and medial prefrontal cortex. J Comp Neurol. 2003; 460:425-449. [PubMed: 12692859]

Petrides M. Impairments on nonspatial self-ordered and externally ordered working memory tasks after lesions of the mid-dorsal part of the lateral frontal cortex in the monkey. J Neurosci. 1995; 15:359-375. [PubMed: 7823141]

Petrides M. Dissociable roles of mid-dorsolateral prefrontal and anterior inferotemporal cortex in visual working memory. J Neurosci. 2000; 20:7496-7503. [PubMed: 11007909]

Petrides M, Alivisatos B, Meyer E, Evans AC. Functional activation of the human frontal cortex during the performance of verbal working memory tasks. Proc Natl Acad Sci USA. 1993; 90:878882. [PubMed: 8430101]

Petrides M, Pandya DN. Dorsolateral prefrontal cortex: comparative cytoarchitectonic analysis in the human and the macaque brain and corticocortical connection patterns. Eur J Neurosci. 1999; 11:1011-1036. [PubMed: 10103094]

Preuss TM. Do rats have prefrontal cortex? J Cog Neurosci. 1995; 7:1-24.

Preuss, TM. What's human about the human brain?. In: Gazzaniga, MS., editor. The New Cognitive Neurosciences. MIT Press; Cambridge: 2000. p. 1219-1234.

Preuss TM. Who's afraid of Homo sapiens? J Biomed Disc Collab. 2006; 1:17.

Previc FH. Dopamine and the origins of human intelligence. Brain Cogn. 1999; 41:299-350.

[PubMed: 10585240] 
Rademacher J, Burgel U, Geyer S, Schormann T, Schleicher A, Freund HJ, Zilles K. Variability and asymmetry in the human precentral motor system. A cytoarchitectonic and myeloarchitectonic brain mapping study. Brain. 2001; 124:2232-2258. [PubMed: 11673325]

Raghanti MA, Stimpson CD, Marcinkiewicz JL, Erwin JM, Hof PR, Sherwood CC. Differences in cortical serotonergic innervation among humans, chimpanzees, and macaque monkeys: A comparative study. Cereb Cortex. 2008; 18:584-597. [PubMed: 17586605]

Raghanti MA, Stimpson CD, Marcinkiewicz JL, Erwin JM, Hof PR, Sherwood CC. Cholinergic innervation of the frontal cortex: Differences among humans, chimpanzees, and macaques. $\mathrm{J}$ Comp Neurol. 2008; 506:409-424. [PubMed: 18041783]

Rizzolatti G, Luppino G, Matelli M. The organization of the cortical motor system: new concepts. Electroencephalogr Clin Neurophysiol. 1998; 106:283-296. [PubMed: 9741757]

Sanides, F. Functional architecture of motor and sensory cortices in primates in the light of a new concept of neocortex evolution. In: Noback, CR.; Montagna, W., editors. The Primate Brain: Advances in Primatology. Vol. Volume 1. Appleton-Century-Crofts; New York, NY: 1970. p. 209-224.

Sawaguchi T, Goldman-Rakic PS. D1 dopamine receptors in prefrontal cortex; involvement in working memory. Science. 1991; 251:947-950. [PubMed: 1825731]

Schmitz C, Hof PR. Recommendations for straightforward and rigorous methods of counting neurons based on a computer simulation approach. J Chem Neuroanat. 2000; 20:93-114. [PubMed: 11074347]

Sesack SR, Bressler CN, Lewis DA. Ultrastructural associations between dopamine terminals and local circuit neurons in the monkey prefrontal cortex: a study of calretinin-immunoreactive cells. Neurosci Lett. 1995; 200:9-12. [PubMed: 8584271]

Sherwood, CC.; Hof, PR. The evolution of neuron types and cortical histology in apes and humans. In: Preuss, TM.; Kaas, JH., editors. The Evolution of Primate Nervous Systems. Evolution of Nervous Systems. Vol. Vol. 4. Academic Press; 2007.

Sherwood CC, Raghanti MA, Stimpson CD, Bonar CJ, de Sousa AJ, Preuss TM, Hof PR. Scaling of inhibitory interneurons in areas V1 and V2 of anthropoid primates as revealed by calcium-binding protein immunohistochemistry. Brain Behav Evol. 2007; 69:176-195. [PubMed: 17106195]

Sherwood CC, Raghanti MA, Wenstrup JJ. Is humanlike cytoarchitectural asymmetry present in another species with complex social vocalization? A stereologic analysis of mustached bat auditory cortex. Brain Res. 2005; 1045:164-174. [PubMed: 15910775]

Shiurba RA, Spooner ET, Ishiguro K, Takahashi M, Yoshida R, Wheelock TR, Imahori K, Cataldo AM, Nixon RA. Immunocytochemistry of formalin-fixed human brain tissues: microwave irradiation of free-floating sections. Brain Res Protoc. 1998; 2:109-119.

Smiley JF, Subramanian M, Mesulam MM. Monoaminergic-cholinergic interactions in the primate basal forebrain. Neuroscience. 1999; 93:817-829. [PubMed: 10473248]

Studler J, Kitabgi P, Tramu G, Herve D, Glowinski J, Tassin J. Extensive co-localization of neurotensin with dopamine in rat meso-cortico-frontal dopaminergic neurons. Neuropeptides. 1988; 11:95-100. [PubMed: 3133572]

Sutoo D, Akiyama K, Yabe K. Quantitative imaging of tyrosine hydroxylase and calmodulin in the human brain. J Neurosci Res. 2001; 63:369-376. [PubMed: 11223911]

van Eden C, Hoorneman E, Buijs R, Matthijssen M, Geffard M, Uylings HBM. Immunocytochemical localization of dopamine in the prefrontal cortex of the rat at the light and electron microscopical level. Neuroscience. 1987; 22:849-862. [PubMed: 3683852]

van Schaik CP, Pradhan GR. A model for tool-use traditions in primates: implications for the coevolution of culture and cognition. J Hum Evol. 2003; 44:645-664. [PubMed: 12799157]

Venator DK, Lewis DA, Finlay JM. Effects of parietal dopamine loss in the medial prefrontal cortex on local baseline and stress-evoked extracellular dopamine concentrations. Neuroscience. 1999; 93:497-505. [PubMed: 10465433]

von Bohlen und Halbach, O.; Dermietzel, R. Neurotransmitters and Neuromodulators. Handbook of Receptors and Biological Effects. Second Edition. Wiley-VCH Verlag GmbH \& Co.; Weinheim, Germany: 2006. 
von Economo, C.; Koskinas, GN. Die Cytoarchitektonik der Hirnrinde des erwachsenen Menschen. J. Springer; Wien und Berlin: 1925.

Wang X-H, Levitt P, Jenkins A O’Brien, Murphy EH. Normal development of tyrosine hydroxylase and serotonin immunoreactive fibers innervating anterior cingulate cortex and visual cortex in rabbits exposed prenatally to cocaine. Brain Res. 1996; 715:221-224. [PubMed: 8739642]

Whiten A, van Schaik CP. The evolution of animal 'cultures' and social intelligence. Philos Trans R Soc Lond B Biol. 2007; 362:603-620. [PubMed: 17255007]

Williams S, Goldman-Rakic PS. Widespread origin of the primate mesofrontal dopamine system. Cereb Cortex. 1998; 8:321-345. [PubMed: 9651129]

Winterer G, Weinberger DR. Genes, dopamine and cortical signal-to-noise ratio in schizophrenia. Trends Neurosci. 2004; 27:683-690. [PubMed: 15474169]

Yousry TA, Schmid UD, Alkadhi H, Schmidt D, Peraud A, Buettner A, Winkler P. Localization of the motor hand area to a knob on the precentral gyrus. A new landmark. Brain. 1997; 120(Pt 1):141157. [PubMed: 9055804]

Zilles K, Dabringhaus A, Geyer S, Amunts K, Qu M, Schleicher A, Gilissen E, Schlaug G, Steinmetz H. Structural asymmetries in the human forebrain and the forebrain of non-human primates and rats. Neurosci Biobehav Rev. 1996; 20:593-605. [PubMed: 8994198] 


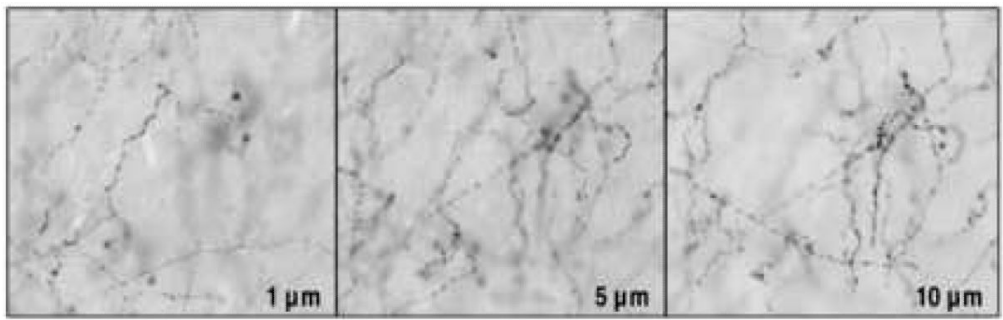

Figure 1.

Examples of TH staining in chimpanzee cortex. High magnification (63x) photomicrographs were captured at $1 \mu \mathrm{m}, 5 \mu \mathrm{m}$, and $10 \mu \mathrm{m}$. 


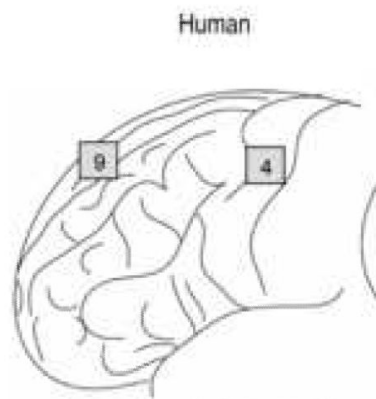

Chimpanzee
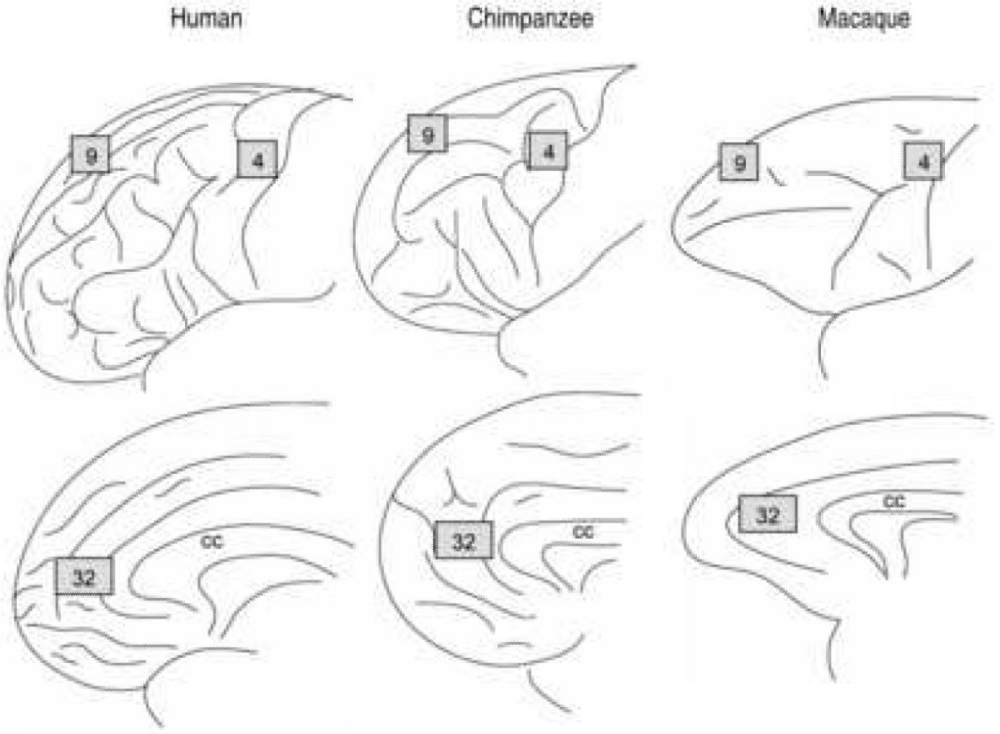

Figure 2.

Lateral (upper) and medial (lower) views of human, chimpanzee, and macaque brains. The positions of the cortical regions sampled in this study are labeled with their respective numerical designations. 

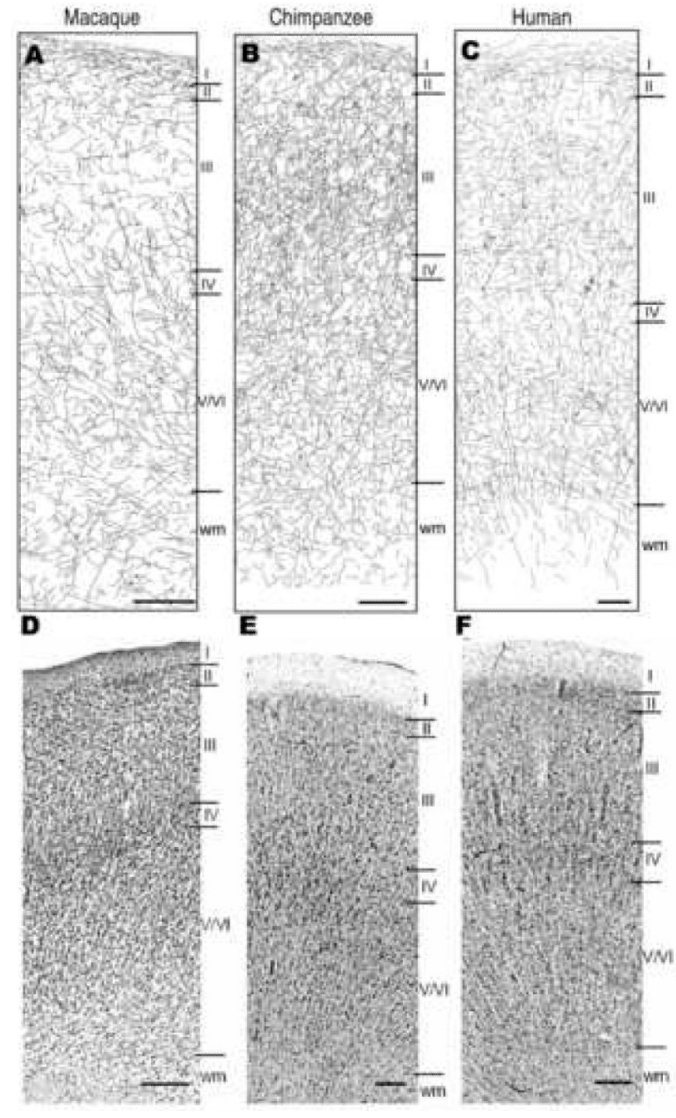

Figure 3.

TH-ir axon tracings (A, B, C) and Nissl-stained sections (D, E, F) in area 9 for each species. Scale bar $=250 \mu \mathrm{m}$. 'wm' $=$ white matter. 


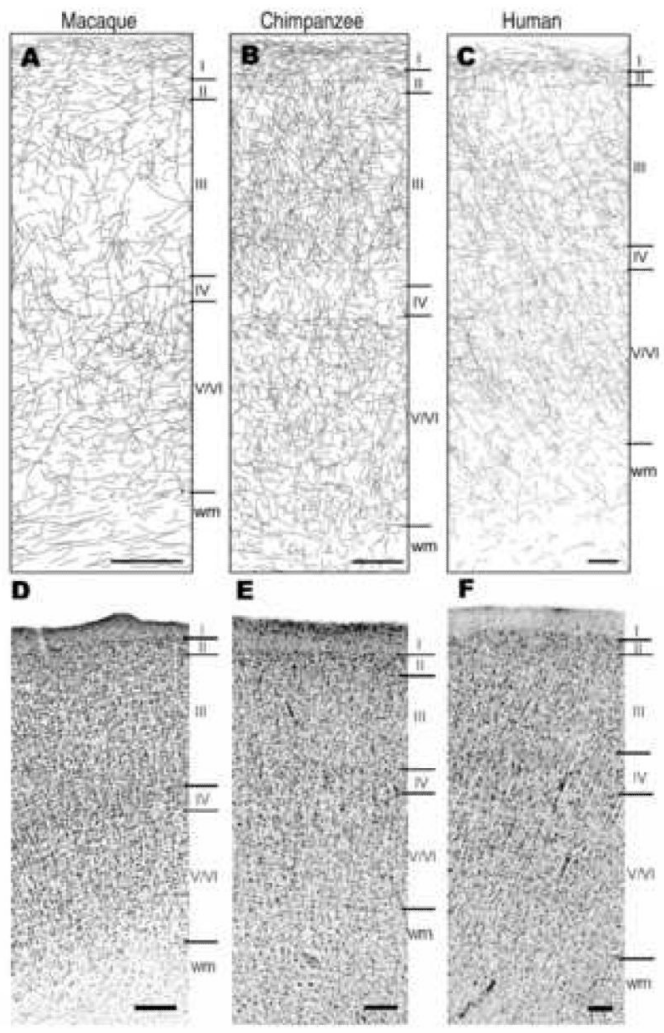

Figure 4.

TH-ir axon tracings (A, B , C) and Nissl-stained sections (D, E, F) in area 32 for each species. Scale bar $=250 \mu \mathrm{m}$. 'wm' = white matter. 


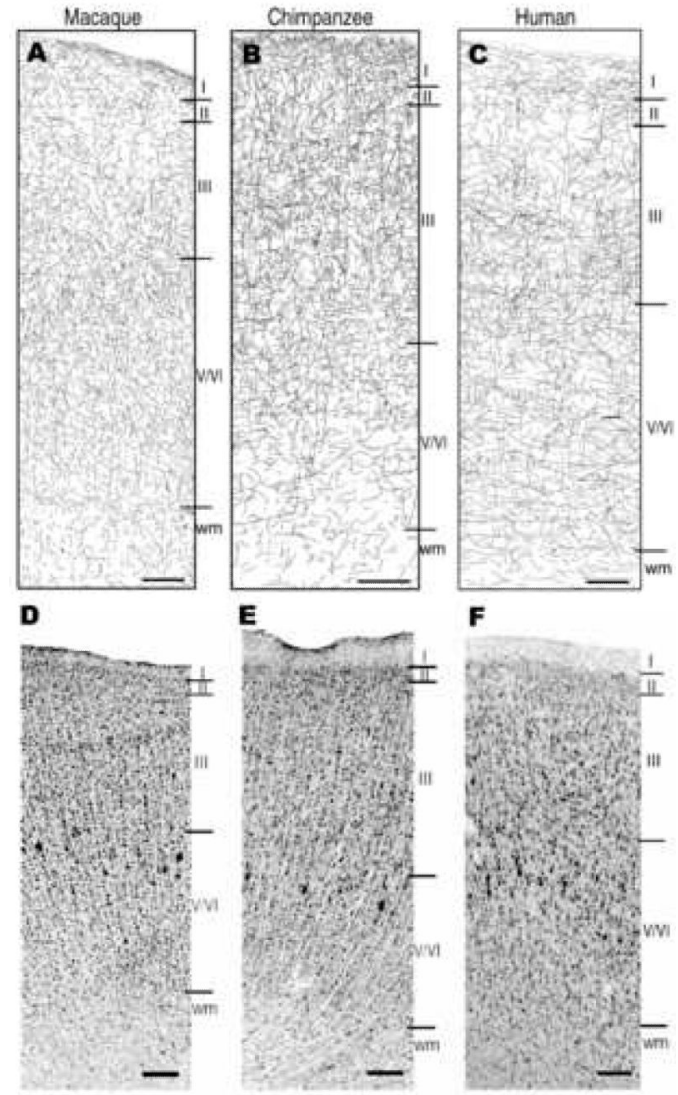

Figure 5.

TH-ir axon tracings (A, B, C) and Nissl-stained sections (D, E, F) in area 4 for each species. Scale bar $=250 \mu \mathrm{m}$. 'wm' = white matter 

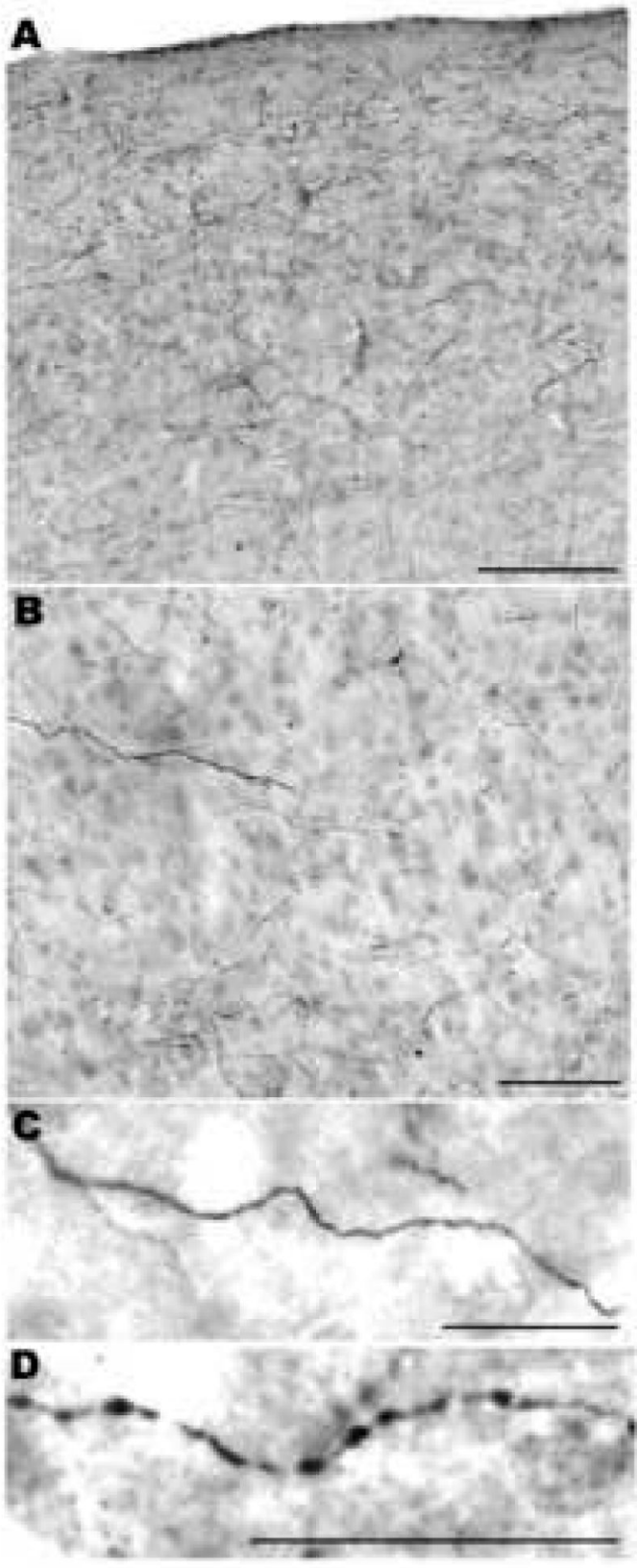

Figure 6.

TH immunoreactivity in layers I-III (A) and V/VI (B) of area 32 in macaque, scale bar $=100$ $\mu \mathrm{m} . \mathrm{C}$ and $\mathrm{D}$ show examples of smooth and varicose axons, respectively, scale bar $=25 \mu \mathrm{m}$. 

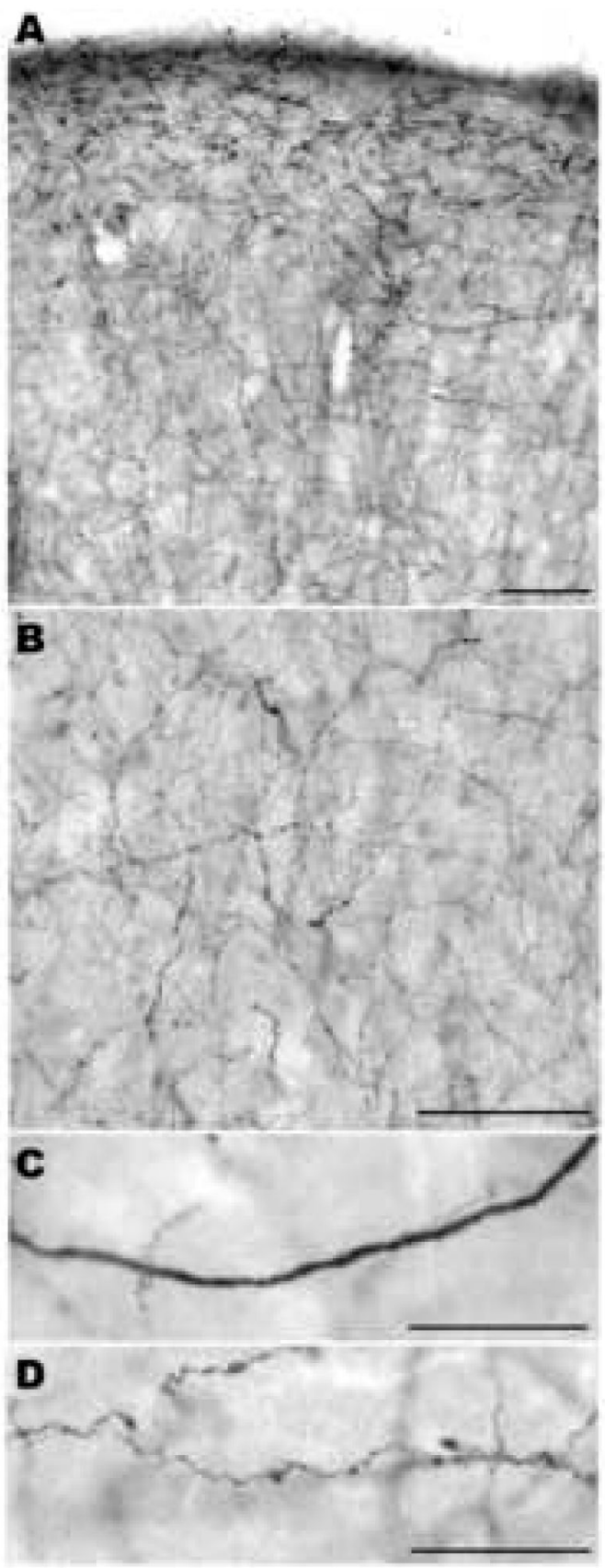

Figure 7.

TH immunoreactivity in layers I-III (A) and V/VI (B) of area 32 in chimpanzee, scale bar = $100 \mu \mathrm{m}$. C and D show examples of smooth and varicose axons, respectively, scale bar $=25$ $\mu \mathrm{m}$. 

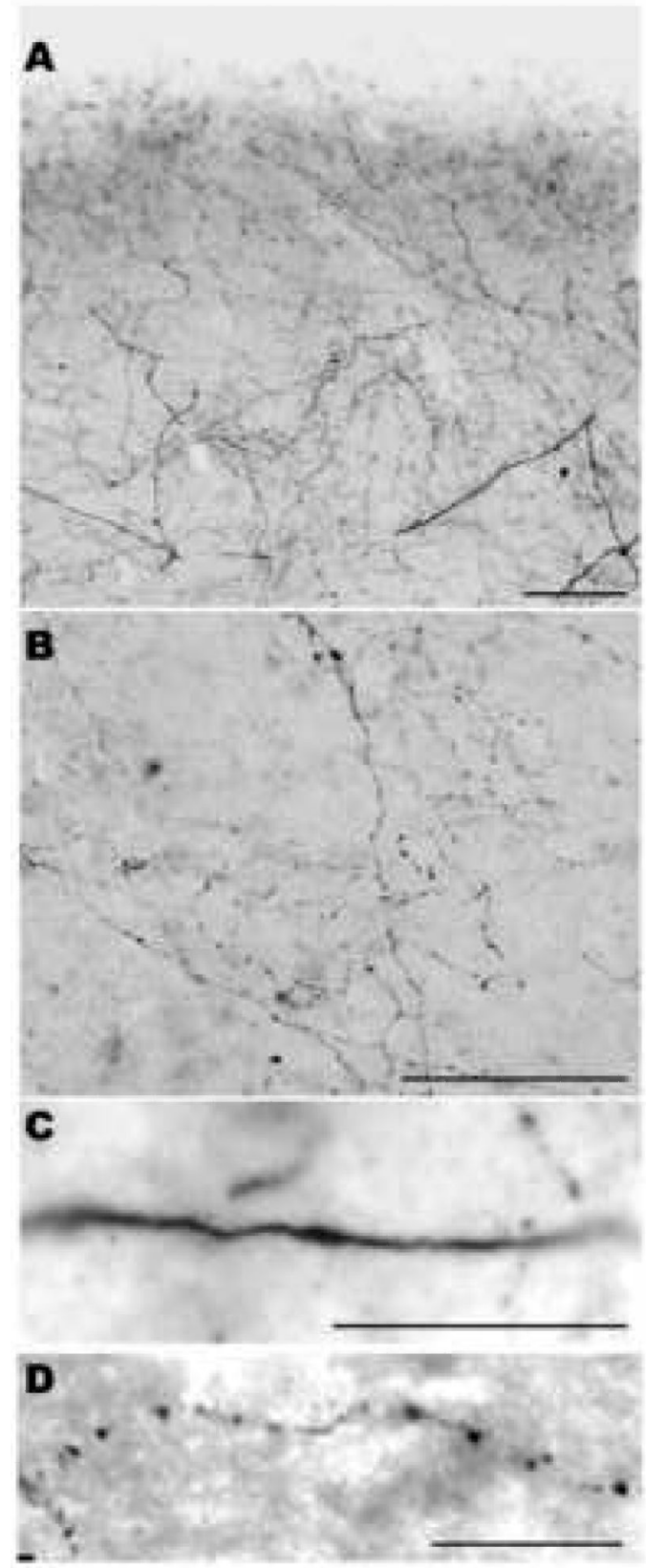

Figure 8.

TH immunoreactivity in layers I-III (A) and V/VI (B) of area 32 in human, scale bar $=100$ $\mu \mathrm{m} . \mathrm{C}$ and D show examples of smooth and varicose axons, respectively, scale bar $=25 \mu \mathrm{m}$. 


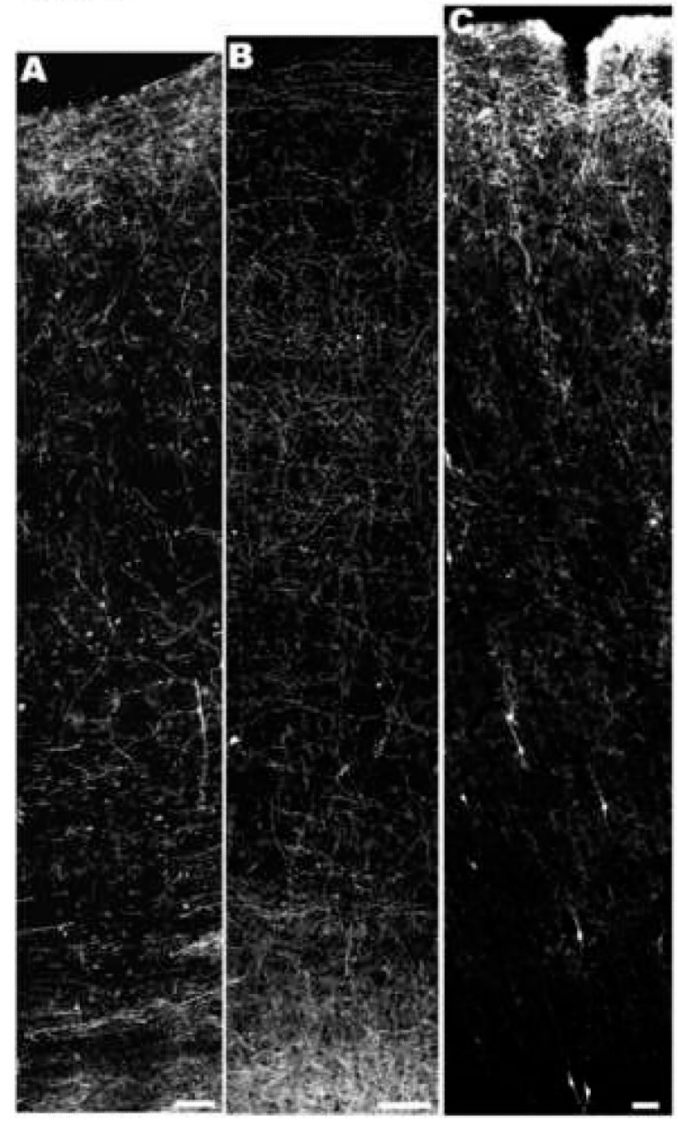

Figure 9.

Darkfield photomicrographs of TH immunoreactivity in macaque (A) and chimpanzee (B). Human area $32(\mathrm{C})$ is a reversed image of a brightfield photomontage. Scale bars $=100 \mu \mathrm{m}$. 


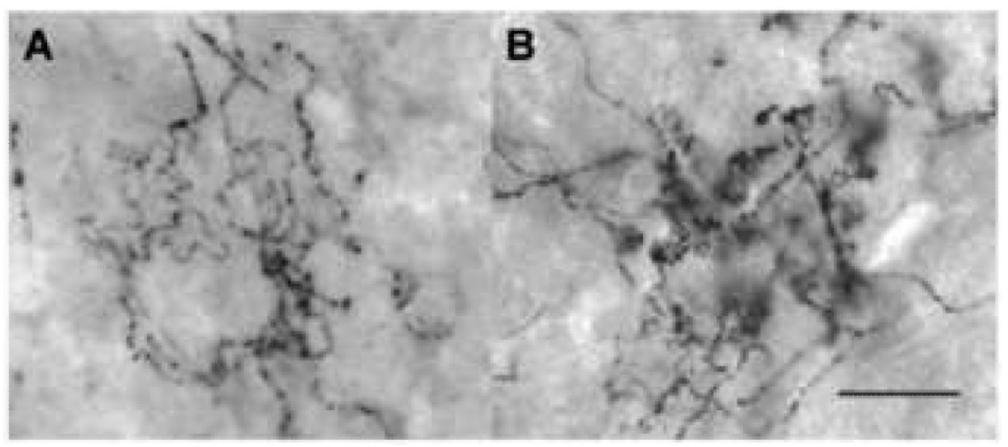

Figure 10.

High magnification photographs of TH-ir coils in human layer III of area 32 (A), and in chimpanzee layer III of area 4 (B). Scale bar $=25 \mu \mathrm{m}(B)$. 

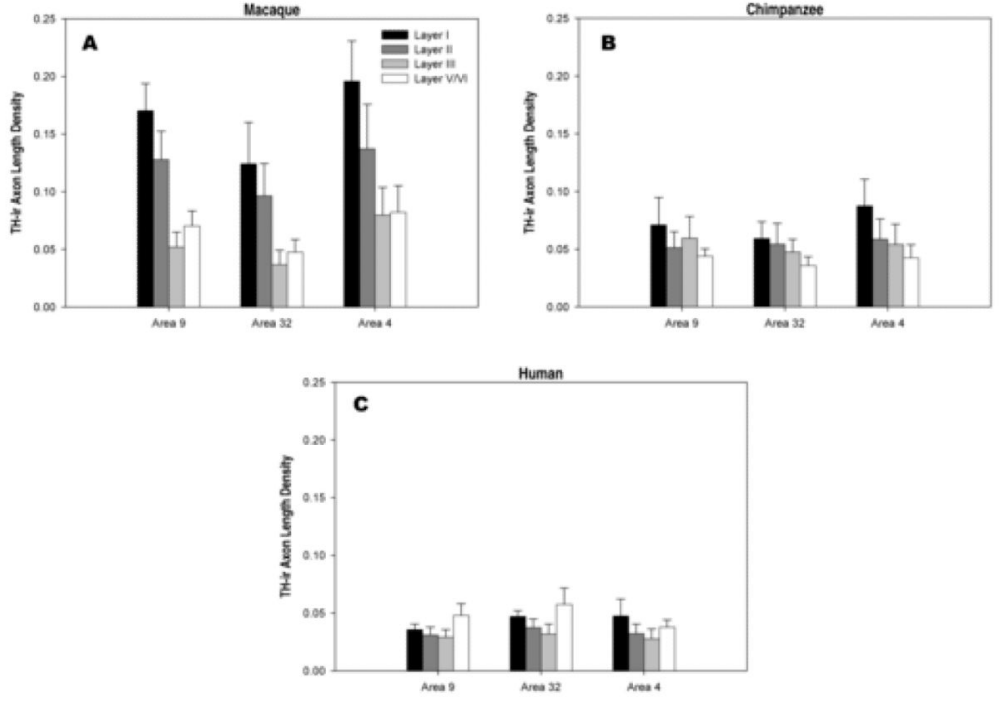

Figure 11.

TH-ir axon length densities in each layer, area, and species. 

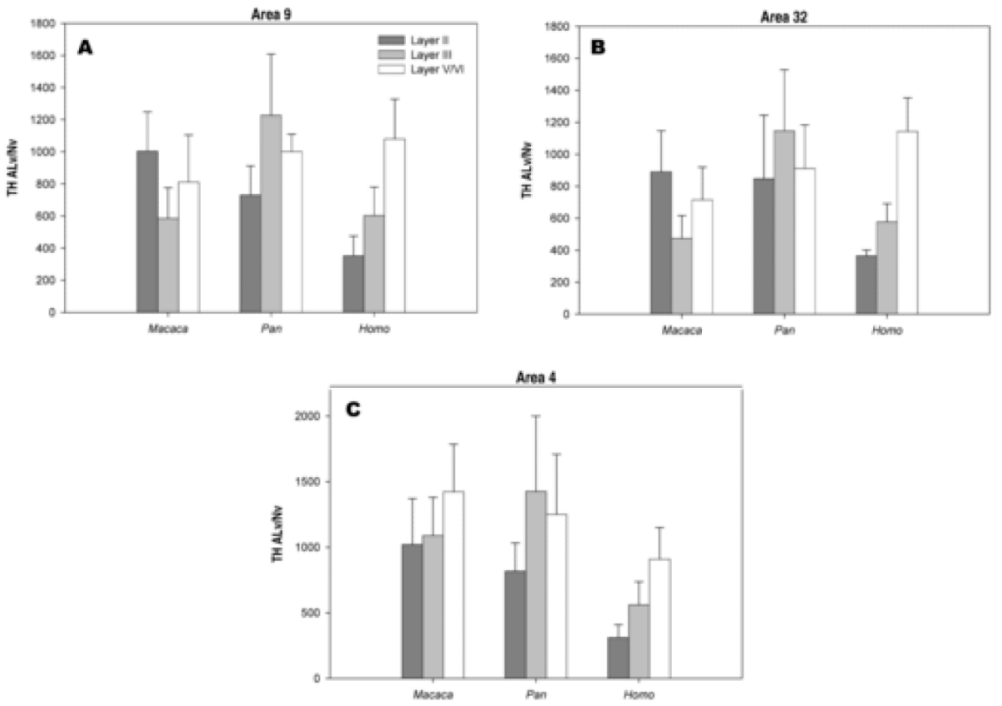

Figure 12.

$\mathrm{TH}$-ir ALv/Nv in each layer, area, and species. 


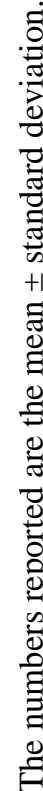

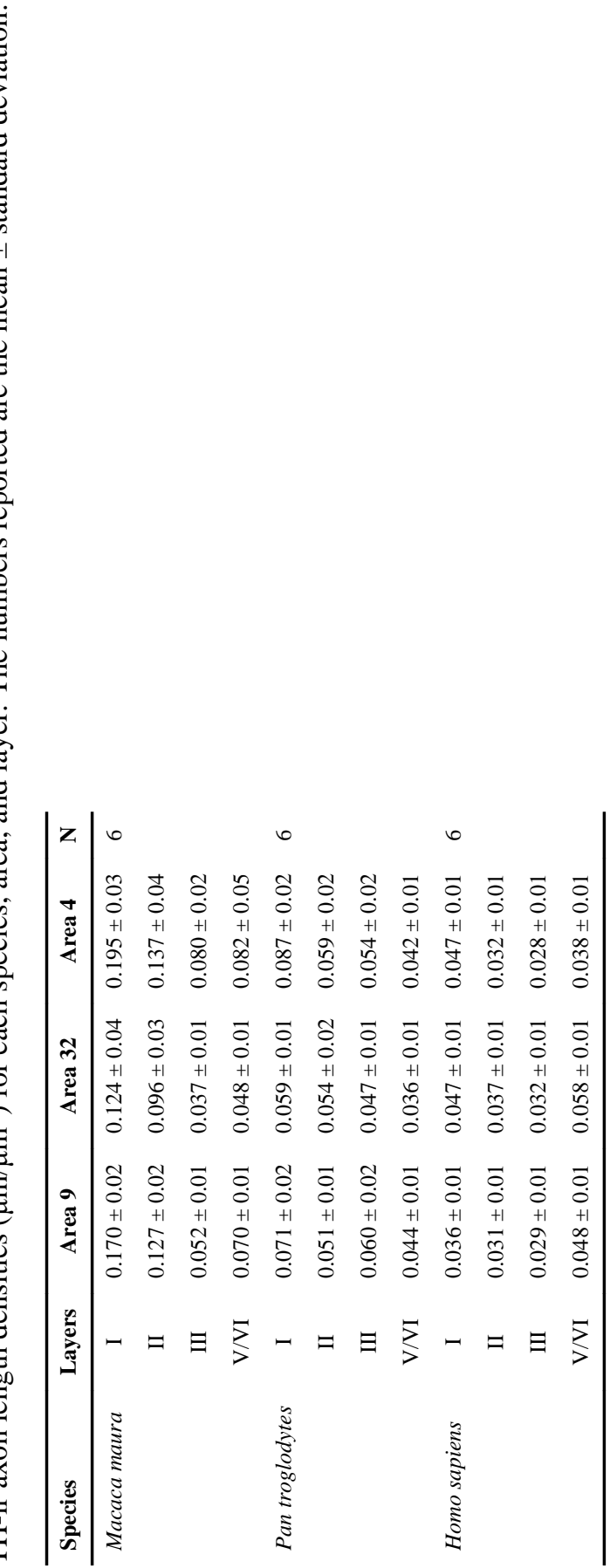


Table 3

Probabilities for Tukey HSD post hoc tests of TH-ir axon length density. Comparisons made between layers within each cortical area for each species.

\begin{tabular}{|c|c|c|c|c|}
\hline Species & Layers & Area 9 & Area 32 & Area 4 \\
\hline \multirow[t]{6}{*}{ Macaca maura } & I-II & $0.00 *(\mathrm{I})$ & $0.05^{*}(\mathrm{I})$ & $0.00 *(\mathrm{I})$ \\
\hline & I-III & $0.00 *(\mathrm{I})$ & $0.00^{*}(\mathrm{I})$ & $0.00^{*}(\mathrm{I})$ \\
\hline & $\mathrm{I}-\mathrm{V} / \mathrm{VI}$ & $0.00 *(\mathrm{I})$ & $0.00^{*}(\mathrm{I})$ & $0.00^{*}(\mathrm{I})$ \\
\hline & II-III & $0.00^{*}$ (II) & $0.00^{*}(\mathrm{II})$ & $0.00^{*}$ (II) \\
\hline & II-V/VI & $0.00 *$ (II) & $0.00^{*}(\mathrm{II})$ & $0.00 *$ (II) \\
\hline & III-V/VI & 0.39 & 0.93 & 1.00 \\
\hline \multirow[t]{6}{*}{ Pan troglodytes } & I-II & $0.04 *(\mathrm{I})$ & 1.00 & $0.00^{*}(\mathrm{I})$ \\
\hline & I-III & 0.61 & 0.58 & $0.00^{*}(\mathrm{I})$ \\
\hline & $\mathrm{I}-\mathrm{V} / \mathrm{VI}$ & $0.00 *(\mathrm{I})$ & $0.00 *(\mathrm{I})$ & $0.00^{*}(\mathrm{I})$ \\
\hline & II-III & 0.91 & 0.98 & 1.00 \\
\hline & II-V/VI & 0.96 & 0.19 & 0.16 \\
\hline & III-V/VI & 0.18 & 0.55 & 0.60 \\
\hline \multirow[t]{6}{*}{ Homo sapiens } & I-II & 0.94 & 0.12 & $0.00^{*}(\mathrm{I})$ \\
\hline & I-III & 0.60 & $0.00^{*}(\mathrm{I})$ & $0.00 *(\mathrm{I})$ \\
\hline & $\mathrm{I}-\mathrm{V} / \mathrm{VI}$ & $0.02 *(\mathrm{~V} / \mathrm{VI})$ & 0.08 & 0.12 \\
\hline & II-III & 1.00 & 0.84 & 0.93 \\
\hline & II-V/VI & $0.00 *(\mathrm{~V} / \mathrm{VI})$ & $0.00^{*}(\mathrm{~V} / \mathrm{VI})$ & 0.84 \\
\hline & III-V/VI & $0.00 *(\mathrm{~V} / \mathrm{VI})$ & $0.00^{*}(\mathrm{~V} / \mathrm{VI})$ & 0.10 \\
\hline
\end{tabular}

Results statistically significant at the 0.05 level. The layer with the higher axon length density is indicated in parentheses to the right of each significant result. 
Table 4

Probabilities for Tukey HSD post hoc tests of TH-ir axon length density. Differences of layers are reported between cortical regions for each species.

\begin{tabular}{lclll}
\hline Species & Layer & Area 9 - Area 32 & Area 9 - Area 4 & Area 32 - Area 4 \\
\hline Macaca maura & I & $0.00^{*}(9)$ & 0.06 & $0.00^{*}(4)$ \\
& II & $0.00^{*}(9)$ & 0.97 & $0.00^{*}(4)$ \\
& III & 0.64 & $0.03^{*}(4)$ & $0.00^{*}(4)$ \\
& V/VI & 0.14 & 0.88 & $0.00^{*}(4)$ \\
Pan troglodytes & I & 0.57 & 0.14 & $0.00^{*}(4)$ \\
& II & 0.99 & 0.96 & 1.00 \\
& III & 0.54 & 1.00 & 0.99 \\
Homo sapiens & V/VI & 0.92 & 1.00 & 0.98 \\
& I & $0.04 *(32)$ & $0.03 *(4)$ & 1.00 \\
& II & 0.70 & 1.00 & 0.89 \\
& III & 1.00 & 1.00 & 0.96 \\
& V/VI & 0.14 & 0.09 & $0.00 *(32)$ \\
\hline
\end{tabular}

Results statistically significant at the 0.05 level. The area with the higher axon length density is indicated in parentheses to the right of each significant result. 
政

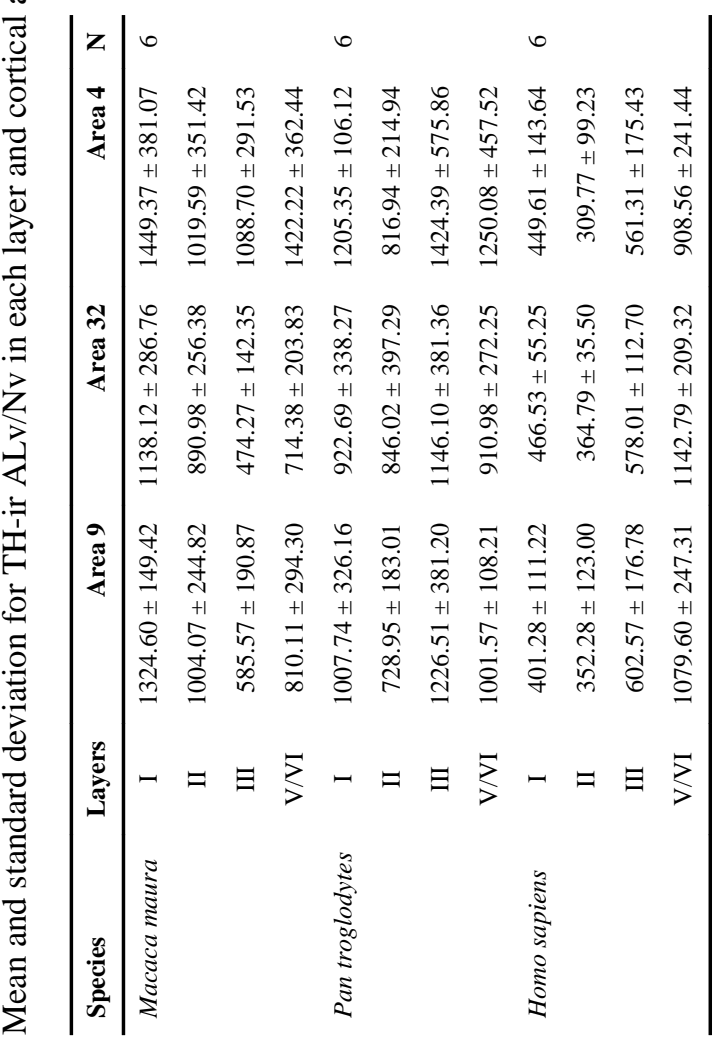


Table 6

Probabilities for Tukey HSD post hoc tests for TH-ir ALv/Nv. Comparisons made between cortical areas.

\begin{tabular}{lcllc}
\hline & Layers & Area 4 - Area 9 & Area 4 - Area 32 & Area 9 - Area 32 \\
\hline Macaca maura & II & 1.00 & 1.00 & 1.00 \\
& III & $0.01^{*}$ & $0.00^{*}$ & 1.00 \\
& V/VI & $0.00^{*}$ & $0.00^{*}$ & 1.00 \\
Pan troglodytes & II & 1.00 & 1.00 & 1.00 \\
& III & 0.99 & 0.70 & 1.00 \\
& V/VI & 0.86 & 0.31 & 1.00 \\
Homo sapiens & II & 1.00 & 1.00 & 1.00 \\
& III & 1.00 & 1.00 & 1.00 \\
& V/VI & 1.00 & 0.91 & 1.00 \\
\hline
\end{tabular}

Results statistically significant at the 0.05 level. 